\title{
Large primary cutaneous angiosarcoma of the nose successfully treated surgically
}

\author{
Kaja Męcińska-Jundziłł¹, Agnieszka Białecka1', Urszula Adamska¹, Arkadiusz Jundziłł², Izabela Neska-Długosz³ \\ Dariusz Grzanka ${ }^{3}$, Henryk Witmanowski², Rafał Czajkowski ${ }^{1}$
}

\begin{abstract}
${ }^{1}$ Clinic of Dermatology, Sexually Transmitted Diseases and Immunodermatology, Faculty of Medicine in Bydgoszcz, Nicolaus Copernicus University in Torun, Poland

${ }^{2}$ Department of Plastic, Reconstructive and Aesthetic Surgery, Medical College in Bydgoszcz, Nicolaus Copernicus University in Torun, Poland

${ }^{3}$ Department of Clinical Pathomorphology, Ludwik Rydygier Collegium Medicum in Bydgoszcz, Nicolaus Copernicus University in Torun, Poland
\end{abstract}

Adv Dermatol Allergol 2020; XXXVII (1): 121-124

DOI: https://doi.org/10.5114/ada.2019.82638

Angiosarcoma is a rare malignant neoplasm. It is a vascular soft-tissue tumour. Cutaneous angiosarcomas most frequently affect elderly people and usually are localized on the head and neck. Clinical presentations differ as erythematous plaques, macules or nodules may be observed, what, in some cases, may suggest benign conditions and the diagnosis is delayed. Histopathological examination is obligatory and the first-line therapy is surgical excision. The prognosis is poor, associated with high rates of local recurrence. We report a case of a 70 -yearold male patient with angiosarcoma of the nose successfully treated with surgical excision in combination with reconstruction of nasal soft tissues using the forehead flap with an aesthetically satisfactory outcome.

A 70-year-old male patient was admitted to our Clinic to diagnose and treat the tumour of the nose. The lesion developed for about 7 months. First the patient observed erythematous macules within skin of the nose, after 3 months the tumour developed.

Before the admission to the hospital the patient was consulted laryngologically. Pus and blood were evacuated from the blister localized within tumour. The patient was treated orally with cloxacillin and topically with chloramphenicol with no improvement. The blister was punctured again but the blood and pus recurred, what suggested infiltration of deeper tissues.

The patient also suffered from hypertension, type 2 diabetes, generalized atherosclerosis and hypercholesterolemia.

On admission to the Clinic the patient presented purple tumour of 4-cm diameter with irregular borders and surface and reduced density in palpation localized on the nose. The lesion involved frontal parts of the nostrils and columella. Within tumour a blister filled with blood and pus was found. Several violaceous, satellite lesions were observed nearby (Figures 1 A, B). The nasal mucosa remained unchanged. In physical examination cervical and submandibular lymphadenopathies were found.

The basic blood laboratory tests showed normocytic anaemia and elevated levels of D-dimers and glucose. Anti-neutrophil cytoplasmic antibodies (cANCA, pANCA) and anti-nuclear antibodies were negative. Abdominal ultrasonography and chest X-ray did not reveal any abnormalities. The computed tomography of the head showed a lesion of $24 \times 17 \times 29 \mathrm{~mm}$ in size within the apex of the nose with an increasing density from $50 \mathrm{HU}$ (Hounsfield units) to $68 \mathrm{HU}$ partially infiltrating cartilages, with no bone tissue involvement. Lesions of soft tissues (approximately $55 \mathrm{HU}$ ), with mild calcifications, filled most of the maxillary sinus without destruction of its bone structures. Besides the paranasal sinuses remained unchanged. Ultrasonography of peripheral lymph nodes confirmed enlarged submandibular, jugular and axillary lymph nodes. Inguinal lymph nodes were normal.

The microscopic examination of nodule biopsy showed extensive infiltration of crowded, small and focally larger, irregular vascular channels. The channels were filled with erythrocytes and lined by atypical endothelial cells with abundant cytoplasm and large, vesicle nuclei with large nucleoli. Focally atypical endothelial cells showed large, hyperchromatic, irregular nuclei. The infiltration was localized subepidermally, involved extensively the dermis and superficially cartilages of the nose (Figures $2 \mathrm{~A}-\mathrm{E}$ ). Based on microscopic examination

Address for correspondence: Kaja Męcińska-Jundziłł MD, PhD, Clinic of Dermatology, Sexually Transmitted Diseases and Immunodermatology, Faculty of Medicine, Nicolaus Copernicus University, 9 M. Skłodowskie-Curie St, 85-094 Bydgoszcz, Poland, phone: +48 602404 829, e-mail: kaja_cm@wp.pl Received: 25.09 .2018 , accepted: 4.10 .2018 

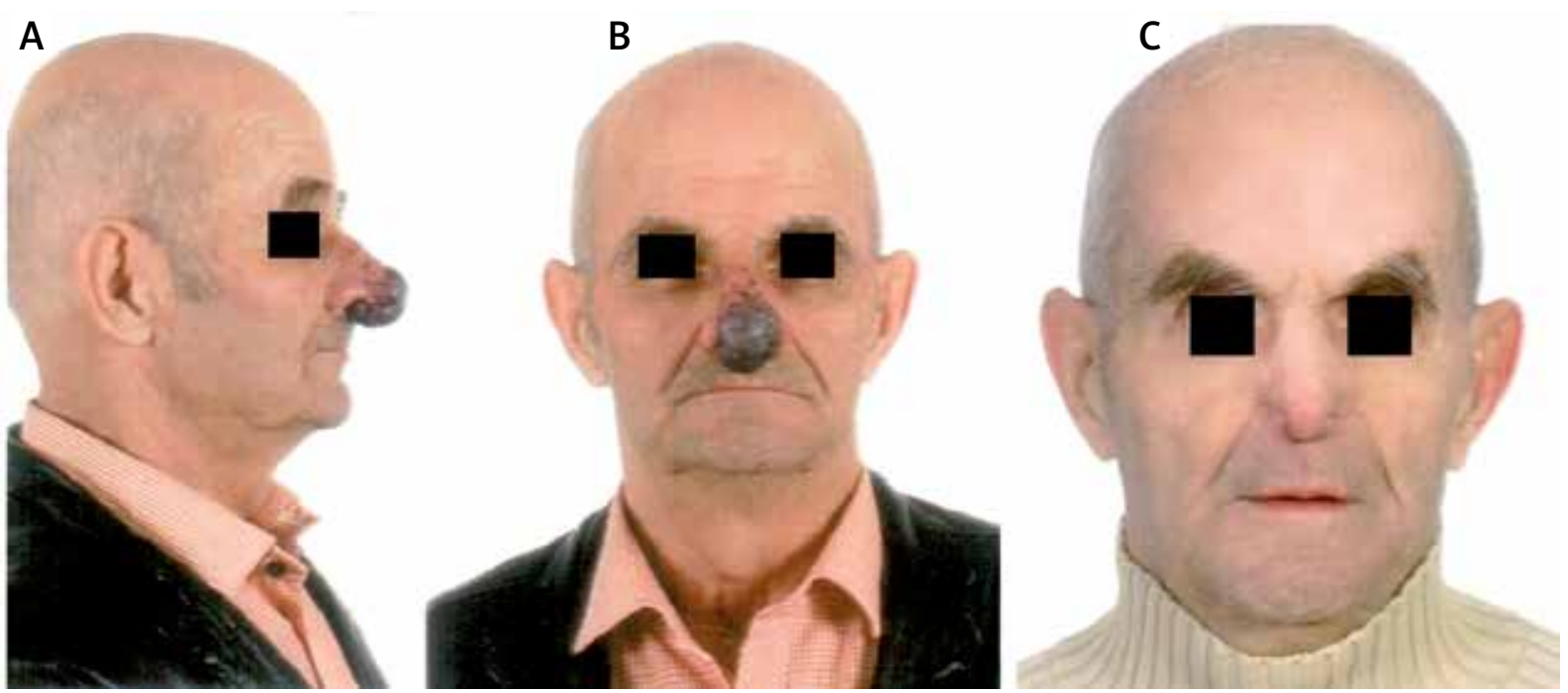

Figure 1. A, B - Purple tumour of 4-cm diameter with irregular borders and surface localized on the nose involving frontal parts of the nostrils and columella with several violaceous, satellite nodules. C - Patient after surgical excision of the tumour and reconstruction of nasal soft tissues with a forehead flap

the diagnosis of G2, pT1 angiosarcoma was established. The patient was referred to the Department of Plastic, Reconstructive and Aesthetic Surgery for treatment. The tumour was excised and the nasal soft tissues were reconstructed using the forehead flap. After 3 weeks the pedicle of the flap was cut off. In the next stage, after 3 months, the patient was hospitalized again to improve the shape of the flap. At that time a small erythematous macula was observed within the skin of the nose suggesting recurrence of the tumour. The lesion was excised and the flap was improved (Figure $1 \mathrm{C}$ ). The histopathological examination of the lesion revealed subcutaneous infiltration of crowded, small vascular channels lined with atypical endothelium showing mitotic activity localized in the dermis (Figure 2 F). The immunohistochemical examination showed positive CD31, von Willebrand factor and CD34 markers confirming endothelial aetiology of the lesion (Figures $2 \mathrm{G}-1$ ). On the basis of microscopic and immunohistochemical examinations the angiosarcoma infiltration was recognized. In the next stages the patient was repeatedly hospitalized to treat the overgrowing nasal passages with no signs of recurrence observed over 3 years.

Cutaneous angiosarcoma is a rare neoplasm of soft tissue derived from endothelial cells. It is very aggressive and the prognosis is poor mainly due to the high rate of recurrence. Angiosarcoma is divided into primary sporadic angiosarcoma, mainly affecting elderly people and frequently involving sun-damaged skin of the head and neck, postradiation angiosarcoma and chronic lymphedema-associated angiosarcoma mostly in patients after radical mastectomies and dissection of axillary lymph nodes due to breast carcinoma [1]. In our patient we diagnosed primary sporadic angiosarcoma involving the face, which is consistent with angiosarcoma of the head, the most frequent subtype. The prognosis is poor with a 5-year overall disease survival rate of 15\% [2]. There are multiple factors which may play a role in angiosarcoma development as chronic sun exposure, lymphostasis and ionizing radiation [3]. The mutation of TP53 is debatable.

The clinical manifestations of angiosarcoma vary. Nodules, papules and macules may be observed. In dermatological practice skin lesions can be mistaken with contact dermatitis, rosacea or haemangioma suggesting benign conditions resulting in delayed diagnosis. There are also cases of angiosarcoma mimicking rhinophyma [2, 4, 5]. In the case of our patient, first he noticed the erythematous macules on the nose, what could suggest an early stage of rhinophyma. The differential diagnosis includes skin cancers like basal and squamous cell carcinoma. Histopathologically angiosarcomas are characterized by irregular vascular channels, lined with atypical endothelium, infiltrating the dermis in the form of a network [6]. Immunohistochemistry examination most frequently reveals positive CD34 and CD31 markers and they were positive in our patient [6]. It is remarkable that CD31 is the most well-known and specific immunohistochemical marker of endothelial cells differentiation among vascular tumours [7]. The first-line therapy is surgical excision, but in some cases when surgery is too extensive resulting in large deformations, there are two chemotherapeutic drugs available (paclitaxel or doxorubicin) [8].

Although there are no strict guidelines concerning width of surgical margins, obviously histopathologically clear margins ensure lower frequency of recurrence [6]. The nasal reconstruction is the most challenging facial reconstruction as the nose is a multidimensional structure localized in the centre of the face [9]. A good aes- 

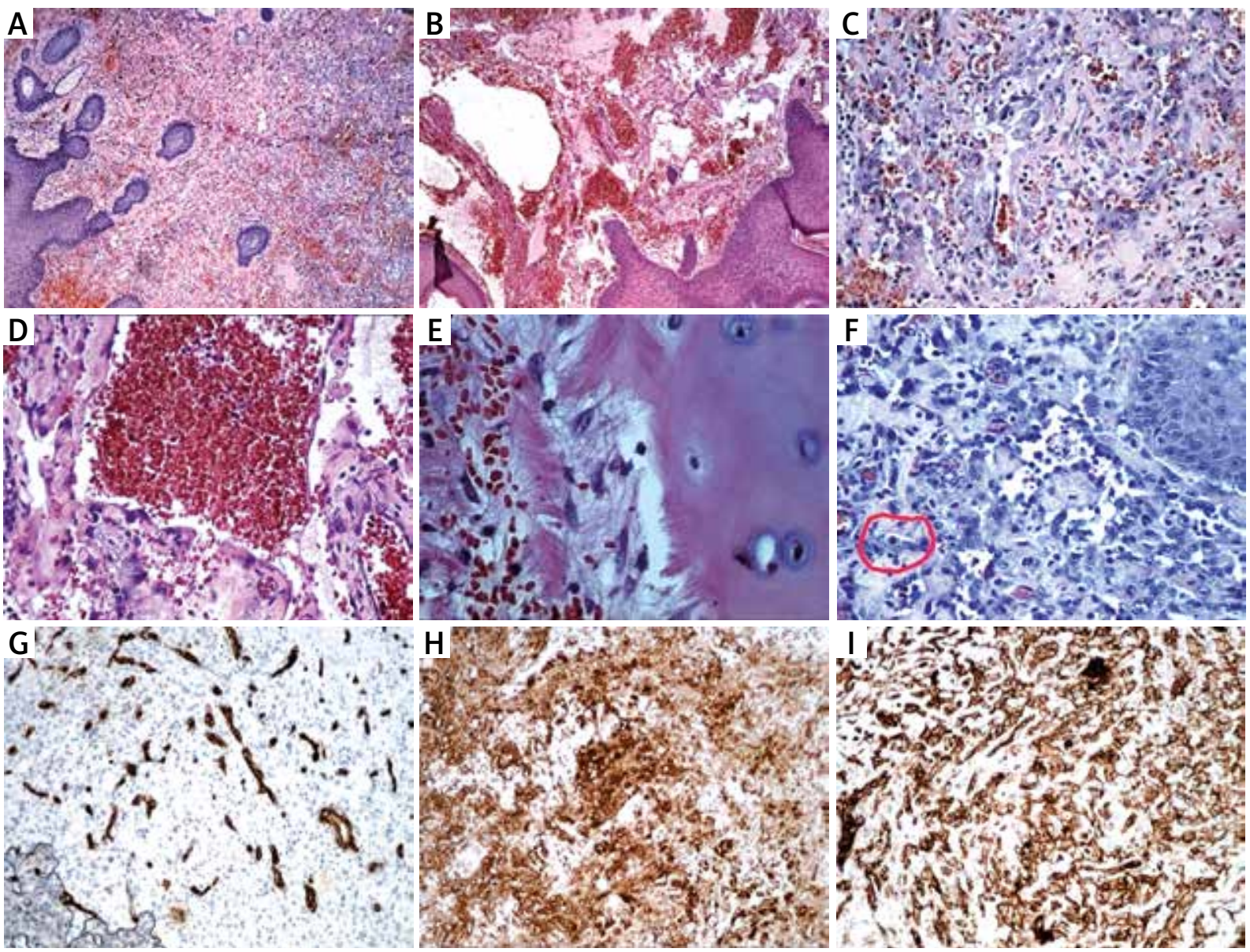

Figure 2. A - The lesion composed of crowded, small vascular channels filled with erythrocytes, localized subepidermally infiltrating the whole dermis. HE, 4×. B - Except small vascular channels, large, irregular, atypical vascular channels filled with erythrocytes are observed. HE, 4×. C - Small vascular channels lined with atypical endothelial cells with abundant cytoplasm and enlarged, vesicular nuclei with pronounced nucleoli. HE, 20x. D - Large vascular channels lined with atypical endothelial cells with abundant cytoplasm and enlarged, hyperchromatic nuclei. HE, 20x. E - Cells of the lesion exceed perichondrium and superficially infiltrate cartilage of the nose. HE, 40x. F - In the second biopsy of the skin, subepidermally localized small vascular channels lined with atypical endothelium. The neoplastic endothelial cell is marked. HE, 20x. G - Immunohistochemistry examination with positive CD34 in atypical endothelial cells within single vessels. IHC, 10x. H - Immunohistochemistry examination with positive von Willebrand factor in atypical endothelial cells. IHC, 10x. I - Immunohistochemistry examination with positive CD31 in atypical endothelial cells. IHC, 20x

thetic result of reconstruction is desirable but functional aspects play an equally significant role. The reconstruction of nasal soft tissues with the forehead flap is the gold standard and this method was used in the case of our patient [9]. The forehead flap delivers a large amount of tissue so that reconstruction of almost any defect is possible [9]. Nasal coverage may be recreated with secondary intention skin grafting, interpolated flaps and local flaps and the method should be selected individually [9]. A staged forehead flap which was used in our case is characterized by best aesthetic results but in patients with relevant comorbidities skin grafts may be more suitable as they require a single operation [9]. In conclusion, angiosarcoma is a rare malignant, vascular tumour of soft tissues. The prognosis is poor because of the high rate of recurrence. The clinical presentation varies and may be mistaken with benign conditions, what delays the proper diagnosis. The first-line therapy is surgical excision and in the case of angiosarcoma involving the nose, the reconstruction using the forehead flap is the gold standard and a staged forehead flap provides the best aesthetic results for the patients.

\section{Acknowledgments}

Kaja Męcińska-Jundziłł and Agnieszka Białecka contributed equally to this work.

\section{Conflict of interest}

The authors declare no conflict of interest. 


\section{References}

1. Shon W, Billings SD. Cutaneous malignant vascular neoplasms. Clin Lab Med 2017; 37: 633-46. 4

2. Aguila LI, Sánchez JL. Angiosarcoma of the face resembling rhinophyma. J Am Acad Dermatol 2003; 49: 530-1.

3. Bergler-Czop B, Hadasik K, Brzezińska-Wcisło L. Clinical and dermoscopic features of atypical abdominal brachytherapyinduced angiosarcoma. Adv Dermatology Allergol 2016; 33: 311-3.

4. Düzgün S, Pekdemir I, Yılancı S, et al. A cutaneous angiosarcoma arising from the rhinophyma. Kulak Burun Bogaz Ihtis Derg 2013; 23: 344-7.

5. Lo Presti M, Mazzella C, Monfrecola A, Falleti J. Angiosarcoma mimicking rhinophyma. Dermatol Res Pract 2010; 2010: 365173.

6. Shustef E, Kazlouskaya V, Prieto VG, et al. Cutaneous angiosarcoma: a current update. J Clin Pathol 2017; 70: 917-25.

7. Ficarra G, Kaban LB, Hansen LS. Central giant cell lesions of the mandible and maxilla: a clinicopathologic and cytometric study. Oral Surg Oral Med Oral Pathol 1987; 4: 44-9.

8. Daguzé J, Saint-Jean M, Dréno B. Large nose angiosarcoma treated effectively with oral cyclophosphamide combined with propranolol. J Eur Acad Dermatol Venereol 2018; 32: e52-4.

9. Correa BJ, Weathers WM, Wolfswinkel EM, Thornton JF. The forehead flap: the gold standard of nasal soft tissue reconstruction. Semin Plast Surg 2013; 27: 96-103. 